\title{
Simulating the interaction between plant roots, soil water and nutrient flows, and barriers and objects in soil using ROOTMAP
}

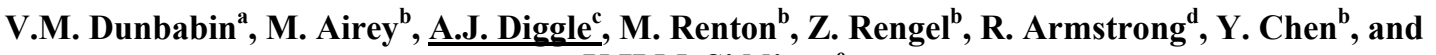 \\ K.H.M. Siddique \\ ${ }^{a}$ Tasmanian Institute of Agricultural Research, University of Tasmania. ${ }^{b}$ The University of Western \\ Australia. ${ }^{c}$ Department of Agriculture and Food Western Australia. ${ }^{d}$ Department of Primary Industries \\ Victoria . ${ }^{e}$ University of Western Australia Institute of Agriculture. \\ Email: art.diggle@agric.wa.gov.au
}

\begin{abstract}
Plant productivity is directly affected by the capacity of the root system to forage for soil resources. An enhanced understanding of root-soil interactions provides the potential to improve crop performance in specific soil environments. Interactions between roots and soil are, however, complex. The root-soil environment is heterogeneous and difficult to visualise and measure, root architecture and root growth responses are complex and dynamic, and processes from the ionic and rhizosphere scale right up to the whole crop and even catchment scale are involved. For these reasons, pot experiments are used in root studies to simplify the environment, target specific interactions and aid with visualisation and measurement. Significant challenges exist, however, in relating pot studies to the field, requiring upscaling from a spatially confined and artificially contrived environment to the reality of a more complex cropping environment. Simulation models provide an opportunity to upscale complex root-soil interactions from the pot to the field, but to do so they must represent the way that plant roots explore a restricted pot environment.
\end{abstract}

In this study ROOTMAP, a 3D functional-structural model of root growth and resource capture, was modified to enable the simulation of barriers in soil, and the interaction of plant roots and soil water and nutrients with those barriers. This barrier-modelling utilises custom coding, with the support of Boost.Geometry (Generic Geometry Library) where appropriate. The barrier approach defines the 3D shape and location of any number of what are termed Volume Objects. Roots and soil can be: wholly contained within one Volume Object such as in the case of roots growing in a pot; a plant can have roots distributed between two Volume Objects such as in a split-pot experiment; and they can be wholly outside one or more Volume Objects for simulating the presence of rocks or other hard objects in soil. Volume Objects can be wholly impermeable, such as; pot walls that contain roots within them, or impermeable rocks or hardpan layers that roots grow around. Volume Objects can also have varying degrees of permeability for representing layers or areas in soil that have varying degrees of hardness and varying root penetrability.

In this initial version of the code, barriers or objects can be represented as rectangular prisms, giving flat barrier layers or square or rectangular objects such as root/rhizo boxes, or as cylinders, representing curved pots or smooth curved objects in soil. The barrier modelling code calculates the deflection of a root tip when it intersects a boundary, representing the way that plant roots grow around and along object surfaces. It also calculates the effect of semi-permeable objects in soil on root growth into and around those objects. Water and nutrients are distributed through the soil environment by use of a variable 3D grid of sub-volumes or cells. The water and nutrient routines then search for the presence of a barrier or wall (Volume Object) intersecting each cell and the volume of the cell contained inside/outside the barrier is calculated. This combined with the permeability of the barrier determines the water and nutrient transfer within the cell. The result is a model which can simulate the root, water and nutrient dynamics in a bounded-environment. This provides an opportunity to represent root architectural development and root-soil interactions in pots and rhizo-boxes, and investigate how these studies relate to root growth and resource capture in un-bounded field soil.

Keywords: ROOTMAP, root modelling, barrier modelling, rhizo boxes 


\section{INTRODUCTION}

ROOTMAP is a three-dimensional (3D) root architecture model that has been designed to simulate the water and nutrient uptake, and root growth dynamics of crop root systems, in response to the changing soil environment (Diggle, 1988; Dunbabin et al., 2002a). Root growth in the model is driven by feedback between plant demand for belowground resources (nitrogen, phosphorus and water), and the capacity for individual root segments to supply those resources. This feedback mechanism determines the allocation of assimilates to root tips for growth, and to all root segments for branching, maintenance and nutrient uptake. The acquisition of soil resources by the root system at any point in time determines the future potential for growth. Using this approach the model can represent whole root system responses to the resource supply as well as localised nutrient uptake and root proliferation responses to local nutrient patches (Fig. 1; Dunbabin et al., 2002a).

ROOTMAP has been used to investigate a range of agronomic problems from water and nutrient dynamics at the micro-scale, up to field-scale simulations of crop water and nutrient use over many seasons (Dunbabin et al., 2002b, 2006, 2009). Roots are notoriously difficult to study due to the fact that they are 'hidden' in soil. ROOTMAP has been designed to investigate complex interactions between crop root systems and their below-ground environment. Such studies are difficult, often impossible, to do in real soil, particularly in the field.

Plants grown in pots in controlled glasshouse conditions form an important component of plant science. The ability to control variables such as water addition, temperature, soil conditions and pests, provides an opportunity to produce reliable, repeatable results in which treatment differences can be more easily discerned. This is particularly true of root studies, where root growth can be highly variable between replicates and the accurate extraction of roots from field soil is difficult.

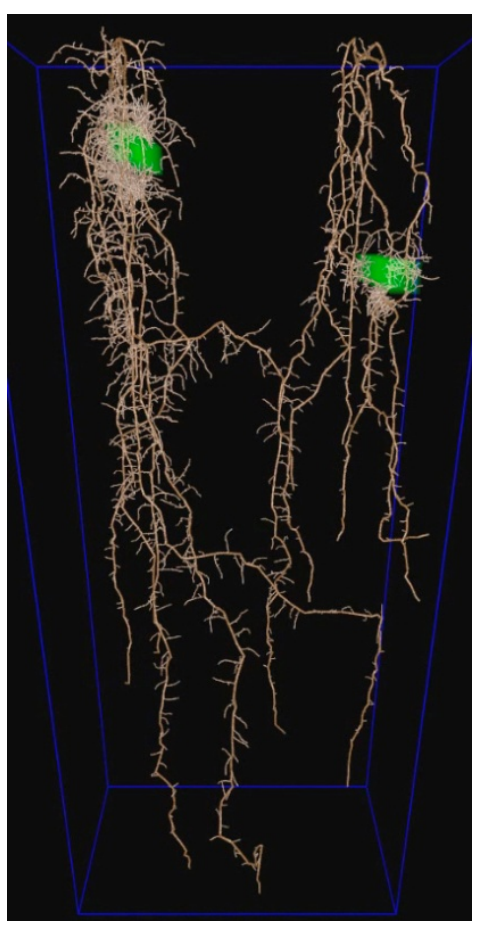

Figure 1. ROOTMAP simulating wheat root systems responding to local phosphorus bands (green) in the soil. With a deeper band (RHS), access to fertilizer P was delayed, giving a smaller root system and less root proliferation around the band.

It can, however, be a significant challenge to extrapolate the results of pot studies to other conditions (eg, other soil types, watering regimes), and to upscale to the field (Passioura 2006). Modelling can play an important role in upscaling from pot to field studies, and in investigating the complexity of root:soil interactions (de Dorlodot et al., 2007). This study extends the ROOTMAP model to represent the interaction between plant roots and objects or barriers in soil. Modifying ROOTMAP to represent objects and barriers in soil provides an opportunity to simulate root growth in pots, including pots with divisions in them for split root experiments, or multiple adjacent pots for simulating split root studies or competition studies. In addition to pot boundaries this approach can represent other obstacles in soil such as rocks, hard pan layers, wax layers in pot studies, or the walls of old growth channels. The model can then be used to upscale to the field.

\section{OVERVIEW OF THE ROOTMAP MODEL}

ROOTMAP is a model of 3-dimensional (3D) root growth, described in detail in Diggle (1988) and Dunbabin et al. (2002a). The model simulates soil water and nutrient dynamics, and root growth responses to those dynamics. ROOTMAP has been developed in $\mathrm{C}++$ with a modular structure. It consists of a number of different modules including the water and nutrient modules, the root architecture module and the resource allocation / root response module. All modules communicate with each other to produce a dynamic root simulator (Dunbabin et al., 2002a). The four key components of the model are; 1.) the engine, 2.) the scoreboard, 3.) the 'plug-in' process modules and 4.) the asynchronous clock.

1. The engine is the central control mechanism that coordinates the behaviour and state of all the other modules. All of the modules communicate with each other via a central message-passing mechanism.

2. The simulation volume is represented by a scoreboard, which is a $3 \mathrm{D}$ rectangular volume, divided into layers in each direction that are not required to be of regular or equal size. The resulting sub-volumes store the local characteristics required by, or created by, each of the process modules (eg. water content, nitrate concentration, root length), and summary information about state variables. This enables both a heterogeneous and dynamic representation of the soil properties. The characteristics of each sub-volume are 
updated each time a process affects the volume, and information can be imported and exported throughout a simulation.

3. The 'plug-in' modules interact with each other via messages. Most of the modules represent plant/soil Processes and are dynamic, having an effect on characteristics in the simulation volume. They require information from other modules as 1D Shared Attributes (stored in the Processes) or as 3D characteristics stored in the scoreboard, and they pass information back for use by other modules and/or the user. Shared Attributes and 3D characteristics represent a common data pool available to the entire simulation.

4. ROOTMAP contains an asynchronous clock that allows each of the modules to operate according to significant events of relevance to that process, at arbitrary times rather than in rigid time steps or scheduling. This allows each of the modules to operate at different time scales and react to events, such as rainfall, that occur at irregular points in time. Individual processes retrieve attributes from the Scoreboard as needed, modify them and reset the new values to the Scoreboard. ROOTMAP is single-threaded, with each process function leaving Scoreboard attributes in a valid state before returning.

The root architecture module (Diggle, 1988), can represent any root architectural arrangement in 3D space, from a simple herringbone structure up to a highly branched, fully dichotomous architecture. A number of parameters define root architecture, including; the number of root orders (or branch orders) that can grow, branch spacing, and the angle branches emerge at (Fig. 2). The direction in which a root tip grows is influenced by the deflection index (the tendency for a root to deflect from its previous heading) and the geotropism index (tendency for root tips to deflect downwards, Fig. 2). ROOTMAP is a stochastic model, hence unique root systems are produced with each model run.

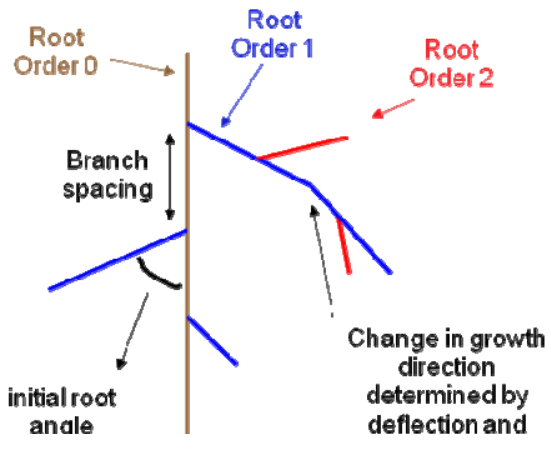

Figure 2. A representation of how root architecture is defined in ROOTMAP Stochasticity is applied to the deflection angle and orientation angle parameters, which then define the unique growth direction of the root tip from a defined region of possible directions. The model can be parameterised with data from pot studies of root architectural traits (Chen et al. 2011), and is valuable for a range of research approaches, including studies of genetic variation in rooting traits aimed at breeding more productive crops (Chen et al. 2011).

Solutes (nitrogen and phosphorus) are transported to the root surface by both massflow and diffusion. The massflow and leaching (via bulk water flow after rainfall) of nitrate are modelled using the Rose et al., (1982) approximate solution to the convection-dispersion equation (Dunbabin et al., 2002a), that utilises truncated normal distributions to calculate the exact 3D coordinate of individual packets (pseudo ions) of nitrate (Diggle 1990). This provides an effective way of visually representing the movement of mobile (nonreactive) solutes through the soil profile.

Solute concentrations at the root surface are derived from local bulk soil values using the Baldwin et al. (1973) model, and plant capacity to take up ions at the root surface is described using Michaelis-Menten kinetics (Dunbabin et al., 2002a). The redistribution of water in 3D space is described by Darcy's law. Pan evaporation, effective crop cover, and local soil properties drive evaporative loss of water from the soil surface (Dunbabin et al., 2009). Along with local root surface area, they also drive the water uptake from each local soil volume, using a Feddes sink term (Feddes et al., 1976). The phosphate routine models the reactivity of the labile phosphate solid-liquid phases across 3D space at each time-step (Mendham et al., 1997; Dunbabin et al., 2009).

\section{MODELLING ROOTS INTERACTING WITH THE SURFACE OF A BARRIER/OBJECT}

The ROOTMAP model was modified to allow it to simulate the interaction between plant roots, water and nutrients, and barriers or objects in the rooting environment. These objects are known as Volume Objects (VOs), and are used to define regions in 3D space with differing soil and permeability properties. A VO can, for example, define the walls of a pot, with roots free to explore the soil within the pot, but deflecting when they interact with a pot wall, representing the way that root systems are bounded in a pot environment (Fig. 3 ). This VO approach can be used to represent a wide variety of object types in soil, such as: barriers in splitpot experiments (impermeable; Fig.4); wax layers in pot experiments used to replicate hardpan layers in the field (permeability can be varied); hardpan layers in field soil (permeability can be varied); rocks or harder areas of soil (impermeable, or varying permeability); and transitions between cracks or root channels and the 
surrounding soil. VO's enable the accurate representation of curved surfaces, hence object shapes are not constrained by the grid of rectangular prisms that defines the Scoreboard.

\subsection{Deflecting roots as they interact with an object}

As roots grow in the model, their direction of growth (heading) is affected by gravity (geotropism), and a deflection index which represents the likely-hood that a root tip will deflect from its current heading (Fig. 2). The barrier code adds an extra component to this calculation, by checking for the presence of barriers or objects (VOs) in soil. If VOs are present, then the model determines where and how the plant roots interact with them. The model iterates over all VOs, and a Deflect Root Segment subroutine determines the type of interaction that each root tip is experiencing (no interaction, interaction with an impermeable VO, interaction with a permeable VO). Most of the time no intersection occurs, so the root grows as a 'normal' unimpeded root. As soon as no intersection is identified, the model skips to the next VO to minimise simulation time. If a root penetrates a VO its heading is not affected, but its growth rate is, since growing conditions change across the penetrated surface. The sub-segment from the intersection point to the end point (root tip) is recalculated, based on the growing-condition variables post-intersection.

If a root interacts with an impermeable object surface, then the Deflect Root Segment subroutine determines the point at which the root tip and the VO intersect, and modifies the trajectory to give a new endpoint for the root tip in that time step, deflecting it around the surface of the VO. Roots still deflect at the soil surface as in previous versions of the model. This barrier version of the code defines two classes of VO: Bounding Cylinder and a Bounding Rectangular Prism. This allows the model to represent cylinders, such as curved pots, soil channels or smooth curved

objects in soil, as well as flat barriers and layers (pot divisions, hardpan layers) or square or rectangular objects such as root/rhizo boxes. Only the interaction between a root segment and a Bounding Cylinder are detailed here.

Deflecting root tips at the surface of a Bounding Cylinder: This routine was written specifically for ROOTMAP and does not contain any third party code. The Deflect Root Segment subroutine uses a start point, a unit heading vector and a magnitude to calculate the new endpoint of a root tip after it has interacted with the surface of a cylinder, causing the root to deflect.

When the bounding object is a cylinder, the root (line segment) can intersect either the curved cylinder wall, or the flat planes at the two cylinder ends. Intersection with the curved wall is checked for first. To do this the parametric line-circle intersection equations (see http://mathworld.wolfram.com/CircleLineIntersection.html) are used to solve for the discriminant ( $\left.\Delta \equiv r^{2} \alpha^{2}-D^{2}\right)$. If $\Delta<0$ there is no intersection with the curved surface; $\Delta=0$, the line is tangential to the curved surface. If it is heading parallel to the direction of axis of the cylinder there is no intersection point. Otherwise there is one intersection point $\left(\mathrm{x}_{\mathrm{i}}, \mathrm{y}_{\mathrm{i}}\right)$, and the parameter $(\mathrm{t})$ value is evaluated accordingly. If $\Delta>0$, the curved surface is intersected, with two possible intersection coordinates $\left(\mathrm{x}_{\mathrm{ia}}, \mathrm{y}_{\mathrm{ia}}\right),\left(\mathrm{x}_{\mathrm{ib}}, \mathrm{y}_{\mathrm{ib}}\right)$. To determine the first intersection point, these coordinates are tested to see which one falls closest to the root segment origin. This is done by using the parametric form of the 2D line equation:

$$
\mathrm{t}_{\mathrm{ia}}=\left(\mathrm{x}_{\mathrm{ia}}-\mathrm{x}_{1}\right) /\left(\mathrm{x}_{2}-\mathrm{x}_{1}\right), \quad \mathrm{t}_{\mathrm{ib}}=\left(\mathrm{x}_{\mathrm{ib}}-\mathrm{x}_{1}\right) /\left(\mathrm{x}_{2}-\mathrm{x}_{1}\right)
$$

If $\mathrm{t}$ is in the range $0<\mathrm{t}<=1$, then the smallest value of $\mathrm{t}$ is the first to intersect the curved surface, otherwise there is no intersection with the curved surface.

For the horizontal component of the root segment, the point $\left(\mathrm{x}_{1}, \mathrm{y}_{1}\right)$ denotes the start of the line segment, and $\left(\mathrm{x}_{2}, \mathrm{y}_{2}\right)$ denotes the end of the line segment (root tip) before being deflected, then we have:

$$
d_{x}=x_{2}-x_{1}, \quad c_{y}=y_{2}-y_{1}, \quad d_{3}=\sqrt{d_{x}^{2}-d_{y}^{2}}, \quad D-\left|\begin{array}{ll}
x_{1} & x_{2} \\
y_{1} & y_{2}
\end{array}\right|-x_{1} y_{2}-x_{2} y_{1}
$$

The points of intersection between the line (root) and the cylinder (of radius $r$ ) are then defined as: 


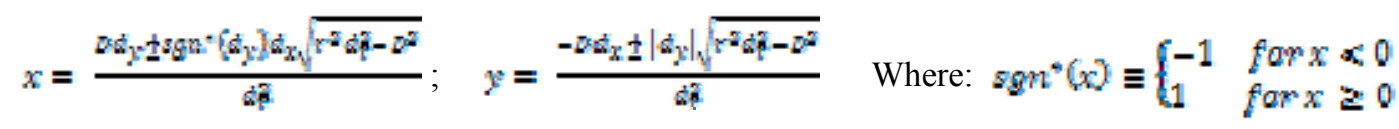

Intersection of the root segment with the top or bottom disc (planes) of the cylinder (line:plane intersection, see http://en.wikipedia.org/wiki/Line-plane intersection) is then checked for. A line intersects a plane at the following point:

$$
i_{\alpha}+\left(\varepsilon_{b}-i_{\alpha}\right) t=p_{2}+\left(p_{1}-p_{b}\right) u+\left(p_{2}-p_{b}\right) v \quad \text { t. } u, v \in \mathbb{R}
$$

Where $\mathrm{l}_{\mathrm{a}}$ and $\mathrm{l}_{\mathrm{b}}$ represent two distinct points $\left(\mathrm{x}_{\mathrm{a}}, \mathrm{y}_{\mathrm{a}}, \mathrm{z}_{\mathrm{a}}\right),\left(\mathrm{x}_{\mathrm{b}}, \mathrm{y}_{\mathrm{b}}, \mathrm{z}_{\mathrm{b}}\right)$ on the line, and $\mathrm{p}_{0}, \mathrm{p}_{1}$ and $\mathrm{p}_{2}$ represent three non-colinear points on the plane. The above can be simplified and expressed in matrix form as:

$$
\left[\begin{array}{l}
x_{Q}-x_{0} \\
y_{Q}-y_{0} \\
z_{\alpha}-z_{0}
\end{array}\right]=\left[\begin{array}{lll}
x_{\alpha}-x_{2} & x_{1}-x_{0} & x_{2}-x_{0} \\
y_{Q}-y_{0} & y_{1}-y_{0} & y_{2}-y_{0} \\
z_{\alpha}-z_{0} & z_{1}-z_{0} & z_{2}-z_{0}
\end{array}\right]\left[\begin{array}{l}
t_{1} \\
w \\
v
\end{array}\right]
$$

Initially only vertical bounding cylinders are considered. This simplifies the above matrix equation, since the points $(0,0, \mathrm{z}),(1,0, \mathrm{z})$ and $(0,1, \mathrm{z})$ are used to define the top plane ( $\mathrm{z}=$ top) and bottom plane ( $\mathrm{z}=$ bottom). Using these points, the inverted matrix may be simplified algebraically. The values of the parameter $t$ for intersection with the top and bottom planes are calculated using the inverted matrix and the vectors from the origin point to the top and bottom planes.

Resulting equation for the top and bottom of the cylinder, $Z_{\text {top/bottom: }}$ :

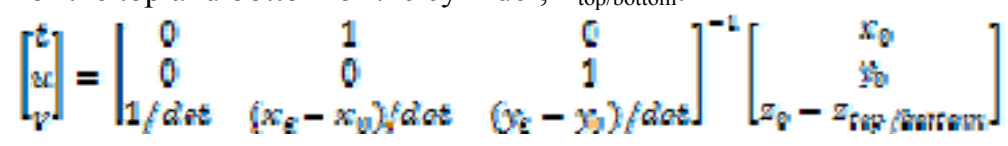

The line segment intersects the top and/or bottom plane if either value of $t$ is in the range $0<t<=1$. If both values of $t$ are in the range $0<t<=1$, the smaller value of $t$ is selected giving the intersection coordinate $\left(\mathrm{x}_{\mathrm{i}}, \mathrm{y}_{\mathrm{i}}\right)$ and the normal vector to this point on the surface is calculated. If both $t$ values (curved surface and top or bottom plane) are invalid, there is no intersection. A valid t value is substituted into the parametric line equation, and the resulting coordinate to ensure that it lies on the boundary of the cylinder and not beyond it (this approach avoids floating point errors), otherwise there is no intersection, and the root continues to grow un-impeded.

For a valid intersection, the coordinate of intersection and the associated unit vector normal to the cylinder surface (radial vector) at that point have now been determined (for the curved surface of the cylinder). Next, the angle between the root segment's heading vector and the vector normal to the intersected surface is calculated. All root segments that deflect at a surface have their intersection coordinate translated along the normal vector by a distance equal to the root radius. This ensures that the root lies against the surface, but not exactly on it, taking into account the effect of the root's thickness on the resulting root geometry. Then, the remaining length of the root segment (the root length after the intersection point) is calculated.

There are then a number of different scenarios depending upon where the intersection occurred: 1.) if the top/bottom disc is intersected, the z-component of the root segment's heading is zero, the heading vector is re-normalised, and the deflected destination point calculated as the intersection point plus a translation of the remaining length along the new heading; 2.) if the intersected surface was curved and intersected from outside the cylinder, the tangential component of the root segment's heading (post-intersection) is deflected into the tangent plane; or 3.) if the curved surface is intersected from inside the cylinder, the root segment is projected onto the curved surface.

The process of deflecting a root tip is then complete. The intersection point (between the root and the surface), the deflected end point (deflected root tip) and the root segment's final heading are returned to the Grow Root Tip routine, and the new segment of root grows. 


\subsection{Water and nutrients interacting with objects}

While the interactions between root growth and surfaces are coordinate-based, water and nutrient transfer is Scoreboard-based (see Overview). Objects in soil (VOs) may impede or restrict transfer of nutrients and water between Scoreboard boxes. VO surfaces may be completely permeable (no transfer restriction), semi-permeable or impermeable (eg. pot walls).

For each Scoreboard box, the model determines whether one or more VOs intersect that box, and calculates the proportion of the volume that is associated with any VO (using a combination of custom coding and the Boost::Geometry computational geometry library - detailed description not given here). This is a computationally intensive process, but only needs to be performed once at the initialisation of a simulation, because neither Scoreboards nor Volume Objects may be modified during a simulation run.

This process then divides each scoreboard box into a number of sub-volumes related to each VO that occupies that box. The scoreboard stores a number of Characteristics that vary in $3 \mathrm{D}$ space (also see Overview), such as soil characteristics (eg. bulk density, drained upper limit, phosphorus buffering index), resource characteristics (eg. water content, nitrogen and

phosphorus content), and plant summaries (eg. root length in each box, water or nutrient uptake from each box). Each of these Characteristics is then divided into a number of sub-Characteristics related to the number of sub-volumes present in each box. That way, each VO in a simulation has its own unique set of Characteristics that define it. Hence, Characteristics vary not only with 3D space, but also with VO (with volume-based characteristics

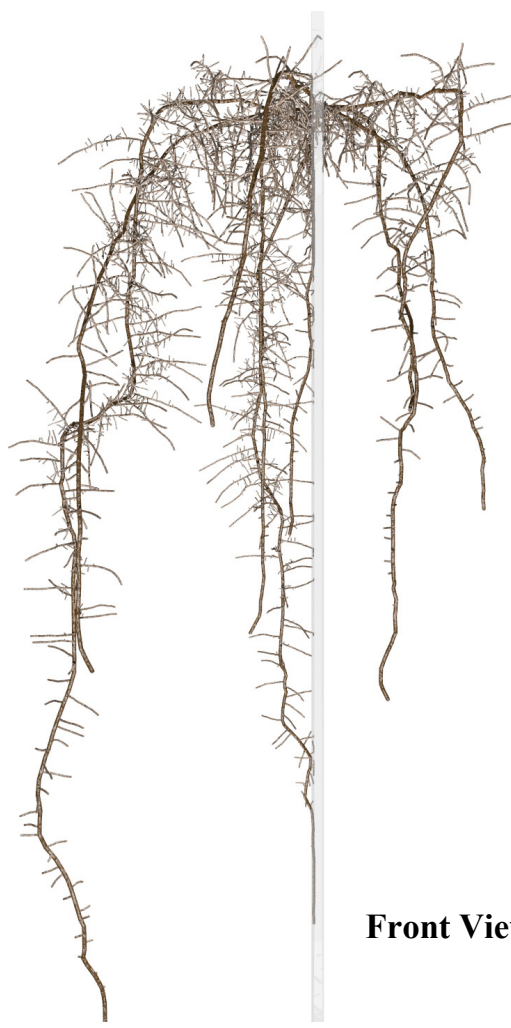

Figure 4. ROOTMAP barrier code simulating a split-pot design. Barrier down the middle of a pot, with dry soil on the right hand side, and reduced root growth on that side. scaling with the intersection volume between the box and the VO). A hardpan layer, for example, will have a different set of soil physical and chemical characteristics associated with it than the remaining soil profile.

The water and nutrient process modules, then take into account not only the $3 \mathrm{D}$ variation in Characteristics as defined by the 3D arrangement of Scoreboard boxes, but they also account for within-box variations in Characteristics brought about by the intersection of a box with a VO. This significantly complicates the processes which transfer water and nutrients between Scoreboard boxes.

The water and nutrient massflow and diffusion routines check whether each Scoreboard box contains subvolumes created by the intersection of that box with one or more VOs. They then check whether the VO is impermeable or semi-permeable (no effect if fully permeable). If impermeable (such as a pot wall), there is no transfer across an impermeable boundary. If semipermeable, then the sub-Characteristics associated with each sub-volume are used to calculate how the nutrients massflow or diffuse through the different parts of that Scoreboard box.

\section{CONCLUSIONS}

Incorporating a structure into ROOTMAP that accounts for objects in the simulation space has considerably expanded the functionality of the model. When growing a new root tip segment, ROOTMAP now checks for the presence of an object in the soil with which the root tip might interact. A root tip may then fully deflect along the surface of that object, such as in the case of a hard pot wall, or an impermeable rock in the soil. A root may penetrate a semipermeable object, such as a hardpan layer, with its
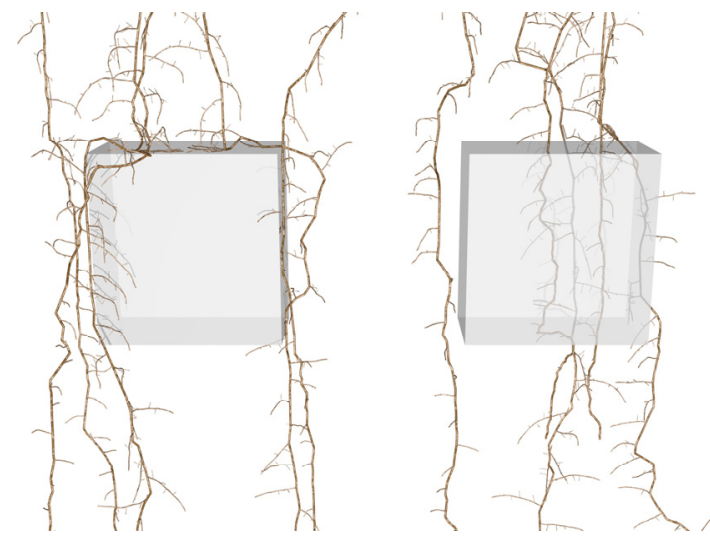

Figure 5. ROOTMAP barrier code simulating two objects, the LHS object is impermeable, and the RHS object is permeable to root growth. 
growth modified by the new conditions in that object. Soil water and nutrient dynamics are also altered by the presence of objects in the soil. This provides an integrated modelling approach for investigating the root growth and water and nutrient flows in response to a range of structures in soil. Pot studies are often used to investigate root:soil dynamics since they provide an opportunity to control environmental parameters, and to more easily extract and measure roots than can be done in field soil. Pot studies are often used to make predictions about root behaviour in the field, and to test and validate simulation models (Liebersbach et al., 2004; Faria et al., 2010). There are, however, challenges associated with using pot studies to represent root and plant behaviour in the field (Passioura, 2006). This is due to a range of factors including the controlled nature of the pot environment and the restriction of root growth which can fundamentally alter the way that plant roots explore and forage for soil resources in 3D space. Equipping ROOTMAP with the capacity to represent bounded soil environments and other objects in soil, provides an opportunity to investigate the interactions between roots and soil in pots, and upscale and investigate the relationship between results from pots and behaviour in the field.

\section{ACKNOWLEDGMENTS}

This work was co-funded by the Australian Research Council and the Grains Research and Development Corporation.

\section{REFERENCES}

Chen, Y.L., Dunbabin, V.M., Postma, J.A., Diggle, A.J., Palta, J.A., Lynch, J.P., Siddique, H.M. and Rengel, Z. (2011). Phenotypic variability and modelling of root structure of wild Lupinus angustifolius genotypes. Plant and Soil, In Press.

de Dorlodot, S., Forster, B., Pagès, L., Price, A., Tuberosa, R. and Draye, X. (2007) Root system architecture: opportunities and constraints for genetic improvement of crops. Trends in Plant Science, 12, 474-481.

Diggle, A.J. (1988). ROOTMAP - a model in three-dimensional coordinates of the growth and structure of fibrous root systems. Plant and Soil, 105, 169-178.

Diggle, A.J. (1990). Interaction between mineral nitrogen and growth of wheat roots in a leaching environment. PhD, The University of Western Australia, Perth.

Dunbabin, V., Diggle, A., Renge,1 Z. and Van Hugten R. (2002a). Modelling the interactions between water and nutrient uptake and root growth. Plant and Soil, 239, 19-38.

Dunbabin, V., Diggle, A. and Rengel, Z. (2002b). Simulation of field data by a three-dimensional model of interactive root growth. Plant and Soil, 239, 39-54.

Dunbabin, V., McDermott, S. and Bengough, A.G. (2006), Upscaling from rhizosphere to whole root system: Modelling the effects of phospholipid surfactants on water and nutrient uptake. Plant and Soil, 283, 5772.

Dunbabin, V.M., Armstrong, R.D., Officer, S.J. and Norton, R.M. (2009). Identifying fertiliser management strategies to maximize nitrogen and phosphorus acquisition by wheat in two contrasting soils from Victoria, Australia. Australian Journal of Soil Research, 47, 74-90.

Faria, L.N., Da Rocha, M.G., De Jong van Lier, Q. and Casaroli, D. (2010). A split-pot experiment with sorghum to test a root water uptake partitioning model. Plant and Soil, 331, 299-311.

Liebersbach, H., Steingrobe, B. and Claassen, N. (2004). Roots regulate ion transport in the rhizosphere to counteract reduced mobility in dry soil. Plant and Soil, 260, 79-88.

Passioura, J.B. (2006). The perils of pot experiments. Functional Plant Biology, 33, 1075-1079.

Rose, C.W., Chichester, F.W., Williams, J.R. and Ritchie, J.T. (1982). A contribution to simplified models of field solute transport. Journal of Environmental Quality, 11, 146-150. 